\title{
RADAR CROSS SECTION CHARACTERIZATION OF CORNER REFLECTORS IN DIFFERENT FREQUENCY BANDS AND POLARIZATIONS
}

\author{
P.V. Jayasri ${ }^{1, *}$, K. Niharika ${ }^{1}$, K. Yedukondalu ${ }^{1}$, E.V.S.Sita Kumari ${ }^{1}$, A.V.V. Prasad ${ }^{1}$ \\ National Remote Sensing Centre, ISRO, Hyderabad, India (jayasri_pv, niharika_k, yedukondalu_k, sitakumari_evs, \\ prasad_avv)@nrsc.gov.in
}

Commission V, SS: Emerging Trends in Remote Sensing

KEY WORDS: Corner Reflector, Radar Cross Section, Trihedral, Dihedral, Compact Antenna Test Range Facility, Calibration

\begin{abstract}
:
Corner Reflectors (CR) are standard passive radar targets which offer one of the best solutions for SAR calibration. Radar Cross Section (RCS) of corner reflectors plays a vital role for estimation of calibration parameters and hence back scatter coefficient for airborne and spaceborne SAR images. There is a stringent requirement to characterize RCS of corner reflectors by measuring its scattering properties in a controlled environment. RCS characterization of square trihedral corner reflectors, dihedrals including polarization selective dihedrals is addressed. These measurements were carried out at $\mathrm{X}, \mathrm{C}$ and $\mathrm{S}$ band frequencies with wide scan angles at definite sampling interval. The design details of corner reflectors, specifications of Compact Antenna Test Range Facility, technical modalities involved for RCS measurements, variation of measured RCS from theoretical value for trihedral and dihedral reflectors at different frequency bands and polarizations are presented in this paper.
\end{abstract}

\section{INTRODUCTION}

For the next few decades, under the category of Advanced Earth Observation Sensors, there are many potential applications of Space borne and Airborne Synthetic Aperture Radar (SAR) images which require the system to be well calibrated in terms of radiometric, geometric and polarimetric calibration (Keyte G.E, 1993). Calibration is a vital exercise for quantifying and qualifying the overall end-to-end system performance including the processing software and ground systems. Before it is announced for scientific and commercial use, the SAR sensor and data processing system have to be well calibrated to ensure accurate and reliable data products. For the calibration and validation of the SAR system, standard radar calibration targets such as Corner Reflectors (CR) are very much essential. The correctness of the calibration exercise depends on the efficacy of the corner reflectors. So there is a need for the characterization of corner reflectors in terms of its Radar Cross Section (RCS) for the transmitted electromagnetic wave.*

Usually, the RCS value of the corner reflector is calculated theoretically by taking into account its shape and dimensions. But the actual RCS of CR may differ from theoretical values due to fabrication errors, if any. Hence, there is a stringent requirement to measure the scattering properties of the corner reflectors in a controlled environment (anechoic chamber) over a range of viewing angles, frequency bands and polarizations.

To carry forward, Square Trihedral and Dihedral corner reflectors along with Polarization Selective Dihedrals of different dimensions are characterized at Compact Antenna test Range Facility.

\section{SAR CALIBRATION FACILITY}

SAR system calibration and validation ensures accurate and reliable data products for effective utilization in remote sensing community. To support Calibration activities during the commissioning and operational phase of Airborne and Spaceborne SAR sensors, a microwave Cal_Val site has been established at National Remote Sensing Centre (NRSC). Conventional Square Trihedral and Dihedral corner reflectors (Figure.1) along with Polarization Selective Dihedrals of different dimensions are designed and deployed in the Cal_Val site to cater to present and future SAR Sensors calibration.

\subsection{Design and development of corner reflectors}

Corner Reflectors (CR) are attractive passive radar targets because of their large bi-static and mono-static Radar Cross Section (RCS) over a wide angular range to perform radiometric, geometric and polarimetric SAR calibration. Square Trihedral Corner Reflector comprises of 3 square aluminium reflecting plates of equal size attached perpendicular to each other. Each panel has a small hole on it to drain rain water and minimizes the effect of strong wind. To perform polarimetric calibration, dihedral corner reflector is designed having two square aluminium plates perpendicularly attached to each other. Fabrication tolerances pertaining to inter-plate orthogonality, plate curvature and plate surface irregularities were specified in order to minimize uncertainties in the expected target response.

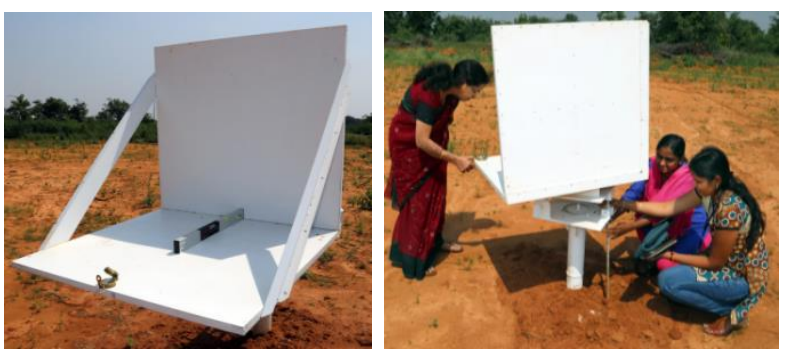

Figure 1: Corner Reflectors deployed at NRSC Microwave CalVal site

\footnotetext{
${ }^{*}$ Corresponding author
} 


\subsection{Deployment of CRs in Cal_Val Site}

The corner reflectors are deployed in Cal_Val site which exhibits low background backscattering and located away from high raised buildings. The position and spacing of CRs is decided based on the resolution of the SAR sensor. These reflectors are oriented in azimuth and elevation directions with respect to bore sight of SAR antenna for each satellite pass.

\section{RCS CHARACTERIZATION OF CORNER REFLECTORS}

Radar Cross Section (RCS) of corner reflectors plays a major role for estimation of calibration parameters and hence sigma naught of the target in the radar image.

\subsection{Radar Cross Section of Corner Reflectors}

Radar scattering is typically represented as the radar cross section (RCS) of the test object. The Radar Cross Section (represented in $\mathrm{dB}$ ) is a measure of the power that is returned or scattered in a given direction, normalized with respect to the power density of the incident field. Basically, RCS of a target is a function of frequency of operation, incident polarization, receiver polarization, target configuration and angular orientation of the target w.r.t the incident field. Accordingly, the RCS of a dihedral and square trihedral depends up on the edge length of $\mathrm{CR}(\mathrm{a})$ and operating wavelength $(\lambda)$ of the sensor. Table 1 represents equations for deriving theoretical RCS values of CRs when its symmetrical axis is aligned with bore sight of the SAR antenna.

\begin{tabular}{|c|c|c|}
\hline $\begin{array}{c}\text { Type of } \\
\text { Corner } \\
\text { Reflector }\end{array}$ & $\begin{array}{c}\text { Radar Cross } \\
\text { Section }(\sigma)\end{array}$ \\
\hline Dihedral & & \\
\hline Srihedral
\end{tabular}

Table 1: Radar Cross Sections of different types of corner reflectors

But practical RCS value of CR may vary from theoretical value because of unavoidable inherent fabrication errors which calls for characterization.

\subsection{RCS Characterization}

The measurement of RCS of a test object requires the test object to be illuminated by an electromagnetic plane wave and the resultant scattered signal to be observed in the far field. It is to be noted that RCS of the test object is a property of the test object alone, and not a function of the radar system or the distance between the radar and the test object, as long as the target is in far field (Borkar V.G., 2010). After calibration, this process yields the RCS of the test object in units of area.

\subsection{Compact Antenna Test Range Facility}

RCS characterization of corner reflector was carried out at Compact Antenna test Range facility (CAR) which is shown in Figure 2. Here, the main purpose of RCS measurement is to collect radar target scattering data at various viewing angles (Garat, J, 1996). The data should correspond to far field where the target is located far enough from the radar so that the incident wave is an acceptably plane wave (knott, E.F, 1974). In Compact range, it uses the reflective properties of a parabolic reflector to correct the phase curvature of electromagnetic wave radiated from a small antenna at the focal point of the reflector. To simulate far-field conditions within a compact range, the reflected wave is collimated and the phase curvature is substantially corrected. The compact range requires a special anechoic chamber having high quality Radar Absorbing Material (RAM) to attenuate reflected electromagnetic energy from the chamber walls (Borkar V.G, 2010).

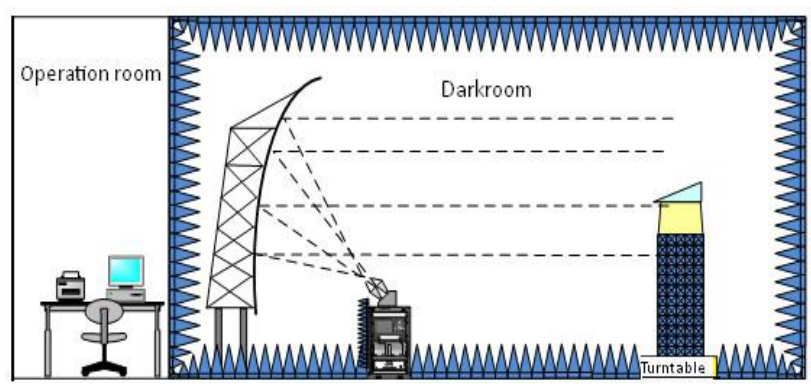

Figure 2: Diagrammatic representation of Compact Antenna Test Range Facility

\subsection{Details of Corner Reflectors Under Test}

Based on technical requirements to conduct RCS measurements for corner reflectors in anechoic chamber at RCI, a wooden supporting structure is designed, fabricated and tested to hold the CRs as shown in Figure 3 at required incident angles.

A test matrix has been prepared for various combinations of frequencies and polarizations along with $\mathrm{CR}$ dimensions and their theoretical values for comparison. Following notation used to represent each corner reflector is based on the shape (Square Trihedral - ST, Dihedral - SD, Polarization selective Dihedrals - SDP) and the numbering is given to represent the uniqueness in that category.

a) Square Trihedrals $(40 \mathrm{~cm}): \mathrm{ST} \_40 \mathrm{~cm} \_3, \mathrm{ST} \_40 \mathrm{~cm} \_4$

b) Square Trihedrals $(75 \mathrm{~cm}):$ ST $75 \mathrm{~cm} \_1, \mathrm{ST} \_75 \mathrm{~cm} \_2$, ST_75cm_3

c) Dihedrals $(60 \mathrm{~cm})$ : SD $60 \mathrm{~cm} \_1, \mathrm{SD} \_60 \mathrm{~cm} \_2$

d) Polarization selective Dihedrals SDP_40cm_HH (SDP1), SDP_40cm_VV(SDP2)

\section{METHODOLOGY}

RCS characterization is implemented by first measuring the characteristics of the target under test (CR) and then taking the measurement data again without the target (termed as background data). The vectorial subtraction of both measurements gives the return contributed only by the corner reflector and its magnitude is a direct measure of its RCS. 


\subsection{Measurement Setup at Test Facility}

For RCS, the measurement requirement is to position the corner reflector whose reflectivity characteristics are to be evaluated on an elevated platform (to be termed as pylon). At the Tx end, two side by side antenna systems, one for transmitting and the other for receiving the backscattered signal, are positioned. Both the antenna systems are physically co-located leading to monostatic measurements. The transmitter generates the RF signal to be radiated through antenna towards the target under measurement. The reflected signal from the target is received by the antenna and goes to the receiver. The receiver detects echo signal that can be stored for further processing or can be recorded by a recorder.

Stepped Frequency Continuous Wave (SFCW) measurement technique is used where a band of frequency is transmitted instead of a single frequency as it carries more information with application of time domain gating which isolates the clutter outside the target zone (Borkar V.G, 2010).
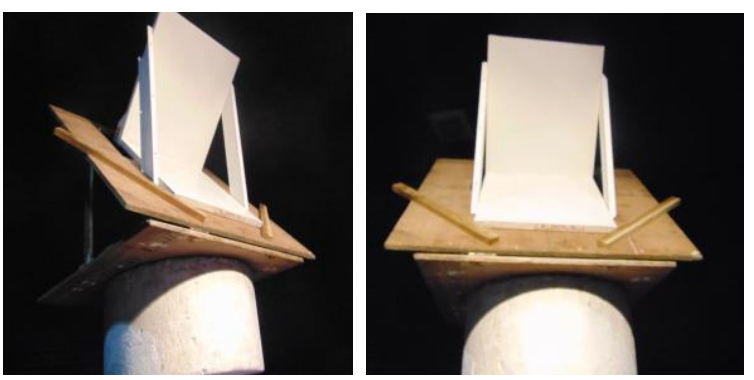

Figure 3: Corner Reflector under test at CAR Facility

Before each measurement in a particular frequency band, the entire test setup is calibrated using different metrological standard targets like flat plates, spheres, discs, cylinders etc. After calibration, Trihedrals \& Dihedrals were mounted on wooden frame at $35.5 \mathrm{deg}$ and $45 \mathrm{deg}$ inclined plane respectively as shown in Figure 3. Based on the provided test matrix, RCS measurements were carried out in different polarizations at $\mathrm{X}, \mathrm{C}$ and $\mathrm{S}$ band frequencies with scan angle of $\pm 60 \mathrm{deg}$ with 0.5 step size (sampling interval) for the corner reflectors.

\section{RESULTS AND DISCUSSIONS}

RCS measurements are primarily performed on Square Trihedrals, Dihedrals and Polarization Selective Dihedrals, the details of which are given in section 3.4. The detailed discussions of the measured results over these corner reflectors are presented in following sections.

\subsection{RCS Measurements for Trihedral Corner Reflectors}

RCS values for two Trihedrals (ST_40cm_3/4) are measured in both VV \& HH polarizations at X-band. It was observed that RCS values are similar for both polarizations, as the three plates of CR are perpendicular to each other. Hence, RCS for other Trihedrals (ST_75cm) are measured only at VV polarization for $\mathrm{S} \& \mathrm{C}$ Band. The measured RCS values in the form of plots are depicted in Figure 4(a) to (f) for trihedral corner reflectors in X, $\mathrm{C}$ and $\mathrm{S}$ bands in co-polarization. Summary of RCS measurements for Trihedral corner reflectors at $\mathrm{X}, \mathrm{C}$ and $\mathrm{S}$ bands in HH and VV Polarization are tabulated in Table 2. It is observed that the variation of measured RCS value w.r.t theoretical value is almost constant for trihedral reflectors for a particular frequency. The deviation from theoretical value can be accounted for fabrication errors and this phenomenon is inevitable in practical scenario.

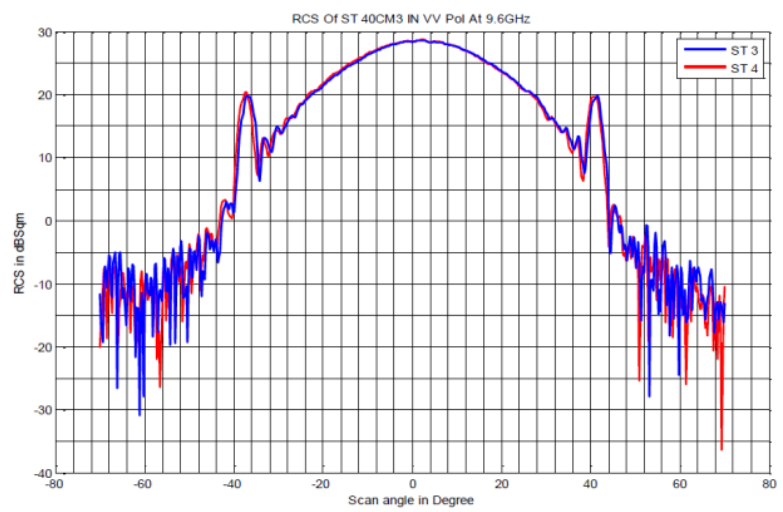

Figure 4(a): Plot of measured RCS values of ST_40cm_3, ST_40cm_4 in VV polarization at X-band $(9.6 \mathrm{GHz})$

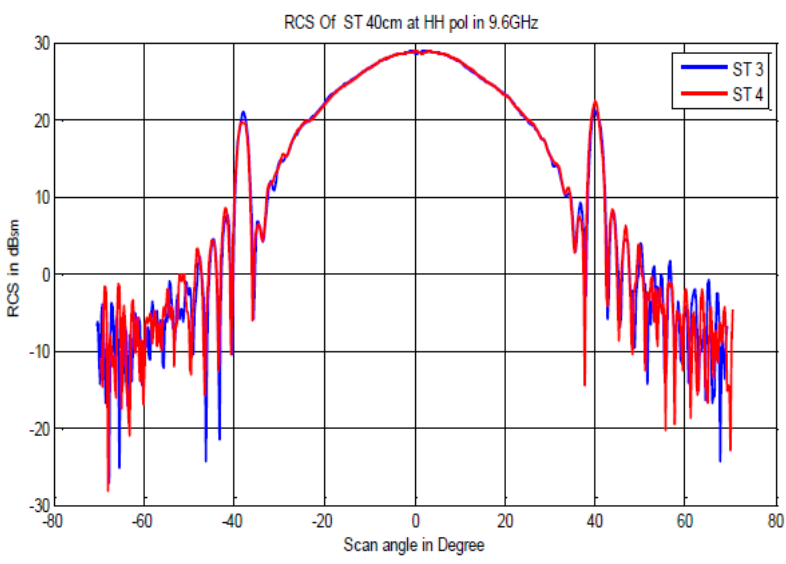

Figure 4(b): Plot of measured RCS values of ST_40cm_3, ST_40 cm_4 in HH polarization at X-band $(9.6 \mathrm{GHz})$

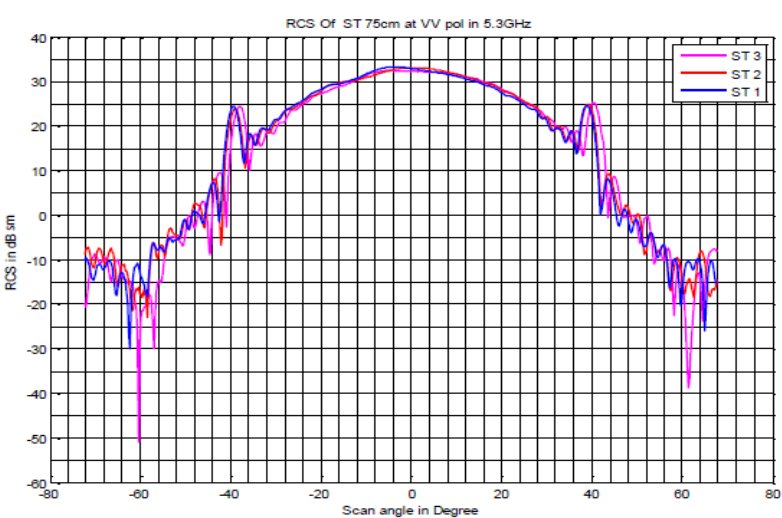

Figure 4(c): Plot of measured RCS values of ST_75cm_1, ST_75 $\mathrm{cm} \_2$, ST_75 $\mathrm{cm} \_3$ in VV polarization at C-band $(5.3 \mathrm{GHz})$ 


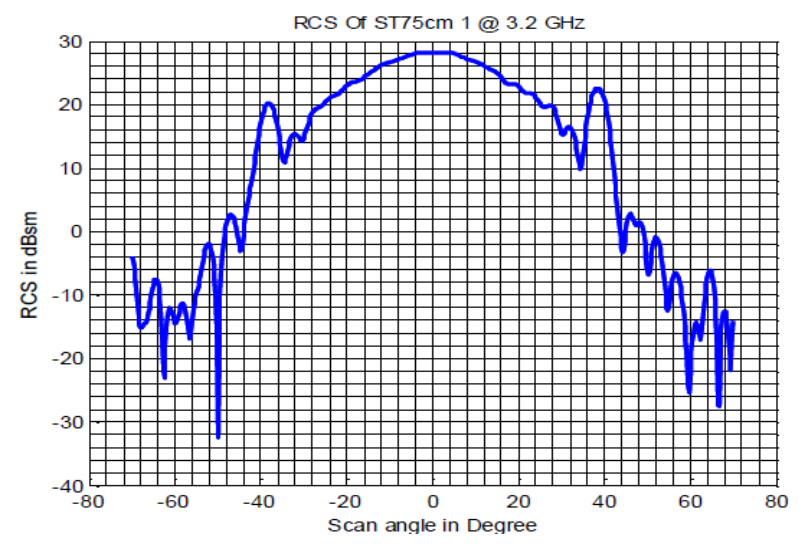

Figure 4(d): Plot of measured RCS values of ST_75cm_1 in VV polarization at S-band $(3.2 \mathrm{GHz})$.

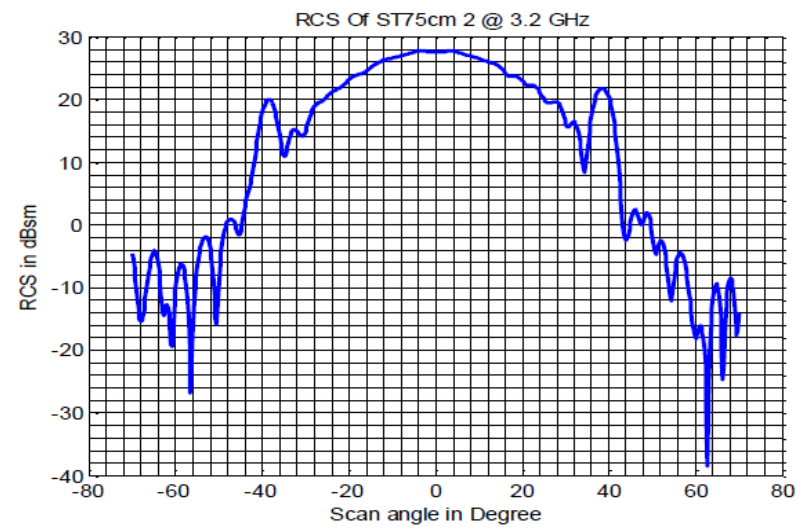

Figure 4(e): Plot of measured RCS values of ST_75cm_2in VV polarization at $\mathrm{S}$-band $(3.2 \mathrm{GHz})$.

\begin{tabular}{|c|c|c|c|c|c|}
\hline $\begin{array}{c}\text { S. } \\
\text { No }\end{array}$ & $\begin{array}{c}\text { CR Notation } \\
\text { Used }\end{array}$ & $\begin{array}{c}\text { Freq } \\
\text { Band, } \\
\text { Polari } \\
\text { zation }\end{array}$ & $\begin{array}{c}\text { Theoret } \\
\text { ical } \\
\text { RCS } \\
(\mathrm{dBsm})\end{array}$ & $\begin{array}{c}\text { Meas } \\
\text { RCS } \\
(\mathrm{dBsm})\end{array}$ & $\begin{array}{c}\text { Differe } \\
\text { nce in } \\
\text { RCS } \\
(\mathrm{dBsm})\end{array}$ \\
\hline ST_40cm_3 & X, VV & 29.57 & 28.6 & 0.97 \\
\cline { 3 - 6 } & X, HH & 29.57 & 28.9 & 0.67 \\
\hline 3 & ST_40cm_4 & XT_VV & 29.57 & 28.8 & 0.77 \\
\cline { 3 - 6 } & X, HH & 29.57 & 28.9 & 0.67 \\
\hline 4 & ST_75cm_2 & C, VV & 35.79 & 33.8 & 1.99 \\
\hline 5 & ST_75cm_3 & C, VV & 35.79 & 33.7 & 2.04 \\
\hline 6 & ST_75cm_1 & S, VV & 31.39 & 28.2 & 3.19 \\
\hline 7 & ST_75cm_2 & S, VV & 31.39 & 27.9 & 3.49 \\
\hline 8 & ST_75cm_3 & S, VV & 31.39 & 28 & 3.39 \\
\hline
\end{tabular}

Table 2: Summary of RCS measurements for Square Trihedral corner reflectors at $\mathrm{X}, \mathrm{C}$ and $\mathrm{S}$ bands

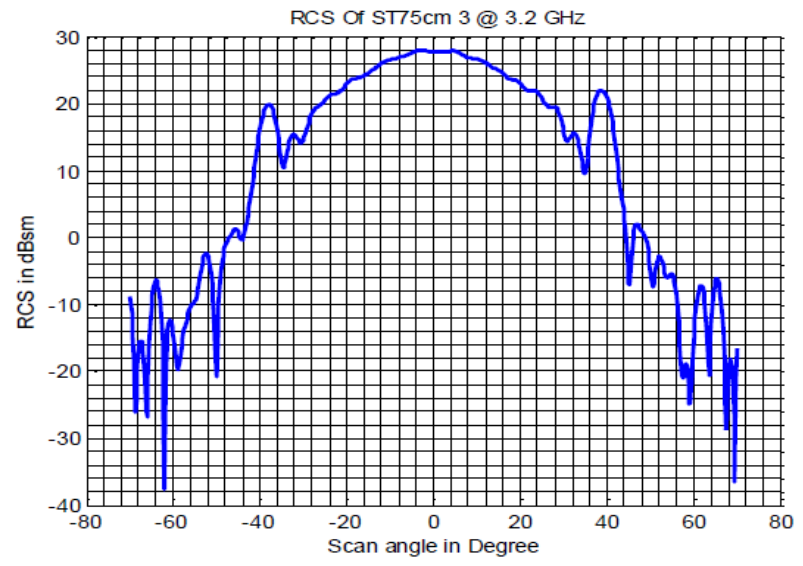

Figure 4(f): Plot of measured RCS values of ST_75cm_3 in VV polarization at S-band $(3.2 \mathrm{GHz})$.

\subsection{RCS Measurements for Polarization Selective Dihedral Corner Reflectors}

RCS values for the Polarization selective Dihedrals (SDP 40cm) are measured in both the polarizations VV \& HH with Dihedrals placed on wooden structure with $45 \mathrm{deg}$ inclination for pointing towards bore sight of antenna.

The RCS plots for polarization selective Dihedrals [SDP $40 \mathrm{~cm} \_$HH, SDP $40 \mathrm{~cm} \_$VV] are shown in Figure 5(a) to (b) measured in both $\mathrm{HH}$ and $\mathrm{VV}$ polarization. These polarization selective dihedrals are having actual dihedral pattern at one of the polarizations and RCS at orthogonal polarization will be 15 to $18 \mathrm{~dB}$ down (Makoto, 2007). Here, SDP_40cm_HH (SDP1) reflector is more sensitive in $\mathrm{HH}$ polarization than in VV and hence more RCS value is observed in $\mathrm{HH}$ polarization. But, as the strips in polarization selective corner reflectors are designed for C-band frequency, little response $(\sim 5 \mathrm{dBsm})$ is observed in VV polarization of X-Band. Same philosophy applies for SDP_40cm_VV(SDP2) when measured in $\mathrm{HH}$ polarization.

\begin{tabular}{|c|c|c|c|c|c|}
\hline $\begin{array}{l}\mathrm{S} \\
\dot{\mathrm{N}} \\
\mathrm{o}\end{array}$ & $\begin{array}{c}\text { CR Notation } \\
\text { Used }\end{array}$ & $\begin{array}{l}\text { Freq } \\
\text { Band, } \\
\text { Polari } \\
\text { zation }\end{array}$ & $\begin{array}{c}\text { Theoret } \\
\text { ical } \\
\text { RCS } \\
\text { (dBsm) }\end{array}$ & $\begin{array}{c}\text { Measure } \\
\text { d RCS } \\
(\mathrm{dBsm})\end{array}$ & $\begin{array}{c}\text { Diff in } \\
\text { RCS } \\
(\mathrm{dBsm})\end{array}$ \\
\hline \multirow{2}{*}{1} & \multirow{2}{*}{$\begin{array}{c}\mathrm{SDP}_{-} 40 \mathrm{~cm}_{-} \\
\mathrm{HH}\end{array}$} & $\mathrm{X}, \mathrm{VV}$ & 0 & 5.1 & 5.1 \\
\hline & & $\mathrm{X}, \mathrm{HH}$ & 27.818 & 21.7 & 6.118 \\
\hline \multirow{2}{*}{2} & \multirow{2}{*}{$\frac{\mathrm{SDP}+40 \mathrm{~cm}}{\mathrm{VV}}$} & $\mathrm{X}, \mathrm{VV}$ & 27.818 & 23.1 & 4.718 \\
\hline & & $\mathrm{X}, \mathrm{HH}$ & 0 & 8.2 & 8.2 \\
\hline
\end{tabular}

Table 3: Summary of RCS measurements for Polarization Selective dihedral corner reflectors at X-band in $\mathrm{HH}$ and VV Polarizations 


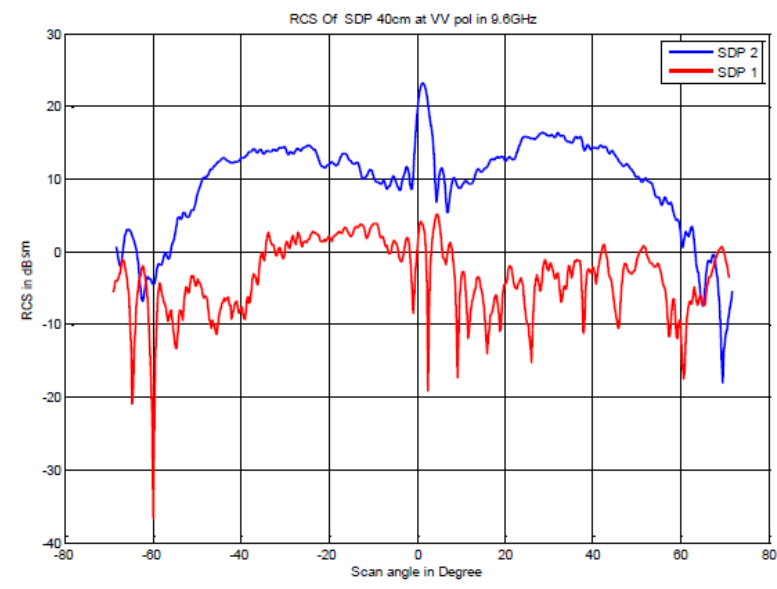

Figure 5(a): Plot of measured RCS values of SDP_40cm_HH, SDP_40cm_VV in VV polarization at X-band $(9.6 \mathrm{GHz})$

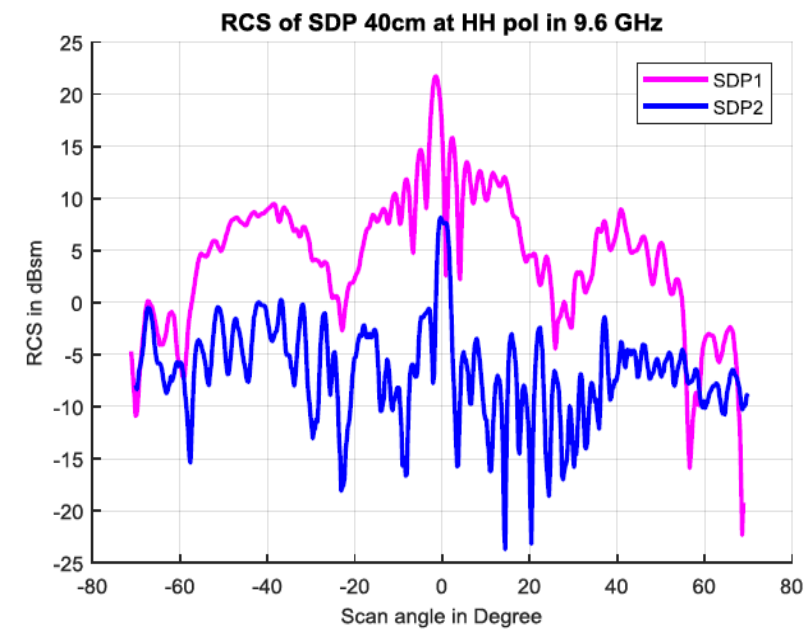

Figure 5(b): Plot of measured RCS values of SDP_40 cm_HH, SDP_40cm_VV in HH polarization at X-band (9.6GHz)

\subsection{RCS Measurements of Dihedral Corner Reflectors}

RCS values of dihedrals (SD_60cm) are measured at S \& C Bands in VV polarization which are tabulated in Table 4. The RCS measurement plots corresponding to Dihedral CRs (SD_60 $\mathrm{cm} \_1$, SD_60 $\mathrm{cm} \_2$ ) at $\mathrm{C}$ and $\mathrm{S}$ band are depicted in Figure 6(a) to 6(c). Ripples are observed in Dihedral patterns, which may be due to supporting wedges joining two faces of dihedrals. The difference in RCS value w.r.t theoretical is due to the support wedges joining two faces of dihedrals which may be obstructing the signal during characterization.

\begin{tabular}{|c|c|c|c|c|c|}
\hline $\begin{array}{l}\mathrm{N} \\
\mathrm{o}\end{array}$ & $\begin{array}{l}\text { CR Notation } \\
\text { Used }\end{array}$ & $\begin{array}{l}\text { Fq. } \\
\text { Ban } \\
\text { d }\end{array}$ & $\begin{array}{c}\text { Theoreti } \\
\text { cal } \\
\text { RCS } \\
(\mathrm{dBsm})\end{array}$ & $\begin{array}{c}\text { Measure } \\
\text { d RCS } \\
(\mathrm{dBsm})\end{array}$ & $\begin{array}{c}\text { Differe } \\
\text { nce in } \\
\text { RCS } \\
(\mathrm{dBsm})\end{array}$ \\
\hline 1 & SD_60cm_1 & $\mathrm{C}$ & 30.1529 & 27.6 & 2.5529 \\
\hline 2 & SD_60cm_2 & $\mathrm{C}$ & 30.1529 & 28.7 & 1.4529 \\
\hline 3 & SD_60cm_1 & $S$ & 25.27 & 21.2 & 4.55 \\
\hline 4 & SD_60cm_2 & $S$ & 25.27 & 20.4 & 5.35 \\
\hline
\end{tabular}

Table 4: RCS measurements for Dihedral corner reflectors at C and $\mathrm{S}$ band in VV Polarization

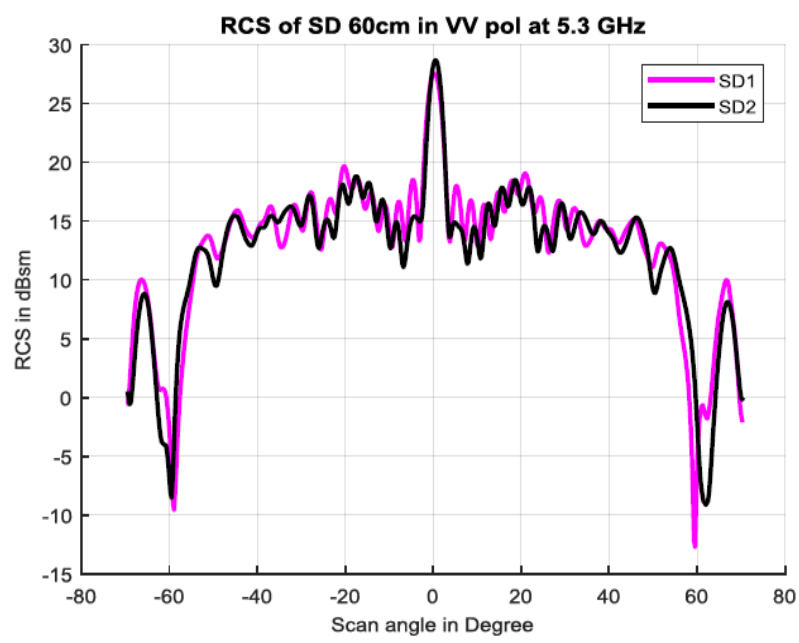

Figure 6(a): Plot of measured RCS values of SD_60 $\mathrm{cm}_{-} 1$, SD_60cm_2 in VV polarization at C-band $(5.3 \mathrm{GHz})$

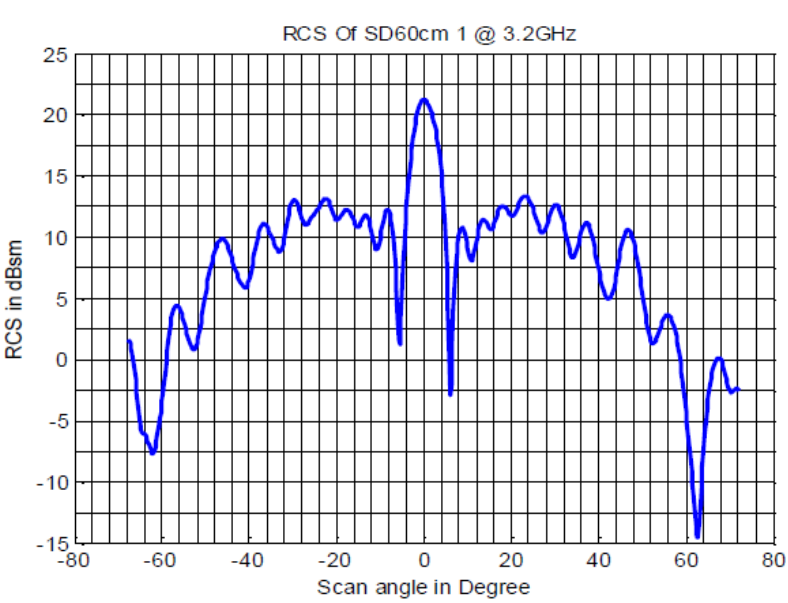

Figure 6(b): Plot of measured RCS values of SD_60cm_1 in $\mathrm{VV}$ polarization at $\mathrm{S}$-band $(3.2 \mathrm{GHz})$

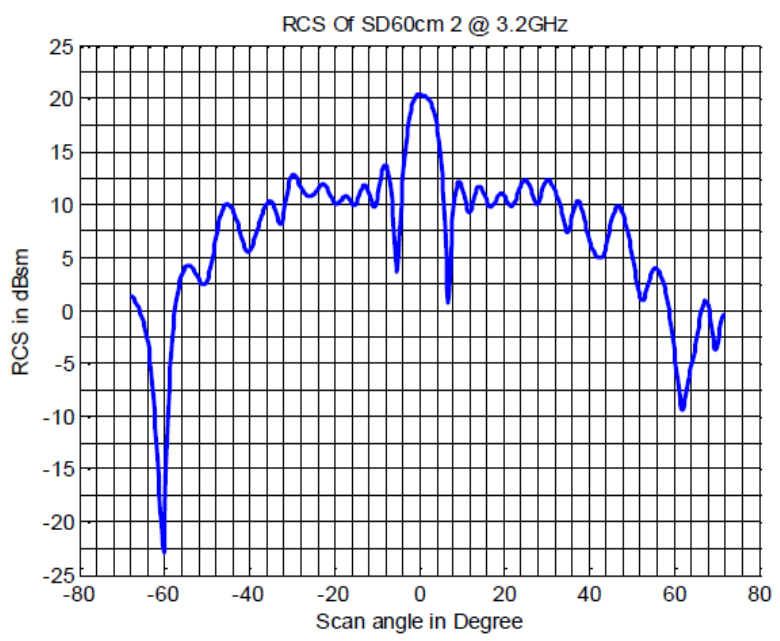

Figure 6(c): Plot of measured RCS values of SD_60cm_2 in VV polarization at $\mathrm{S}$-band $(3.2 \mathrm{GHz})$ 


\section{CONCLUSION}

Radar Cross Section (RCS) of corner reflectors plays a vital role for estimation of SAR calibration parameters. The unknown reduction in the RCS due to fabrication errors will be well ascertained by measuring the RCS of corner reflectors in a controlled environment. Hence RCS characterization of corner reflectors was performed at various frequency bands and polarizations. The technical modalities involved in the execution of RCS characterization are presented in this paper. The measured RCS values of corner reflectors will be useful for the absolute calibration of future SAR sensors.

\section{ACKNOWLEDGEMENTS}

Authors are highly grateful to Shri. Santanu chowdhury, Director, NRSC and Shri Vinod Bothale, DD, DPPA\&WAA for their support to continue this activity. The authors are thankful to Dr. Y.V.N. Krishna Murthy, Ex-director NRSC who gave impetus to initiate this MOU with RCI. The authors are indebted to the technical support provided by Mr. Rakesh Kumar Singh, Scientist 'F' and Mr. Nitin Chourasia, Scientist 'E' RCI, Hyderabad towards use of the experimental set up and guidance during RCS measurement.

\section{REFERENCES}

Borkar V.G., Ghosh. A, Singh R.K., and Chourasia. N, Radar Cross-section Measurement Techniques, Defence Science Journal, Vol. 60, No. 2, March 2010, pp. 204-212

Garat, J. Microwave techniques for radar cross section measurements.A review. In MELECON. 96: Electrotechnical Conference, 13-16 May 1996. pp.80-86.

Keyte G.E., Bird P.J., Kenward D.R.D, Long term radiometric calibration of ERS-1 SAR - Geoscience and Remote Sensing Symposium, 1993. IGARSS-93.

Knott, E.F. \& Senior, T.B.A. How far is far. IEEE Trans. Antennas Propag., September 1974, 732-34.

Makoto Satake, Takeshi Matsuoka, Toshihiko Umehara, Akitsugu Nadai, and Seiho Uratsuka. Development of polarization selective corner reflectors and its experiment for calibration of airborne polarimetric Synthetic Aperture Radar. Proceedings of ISAP2007, Niigata, Japan.

Pravakar Mallick, Milan Kumar pal, Arun Kumar Ray, Raghvendra Kumar Chaudhary, Characterising radar cross section signature for evaluation in test range, DOI: 10.1109/ICMAP.2018.8354535

Ulaby F.T. et al. Microwave Remote Sensing. Volume II, Addison-Wesley, 1982. 